\title{
A smart partial discharge classification SOM with optimized statistical transformation feature
}

\author{
Zul Hasrizal Bohari', M Isa², A Z Abdullah ${ }^{3}$, P J Soh ${ }^{4}$, M F Sulaima ${ }^{5}$ \\ ${ }^{1}$ CeRIA, Faculty of Electrical and Electronic Engineering Technology, Universiti Teknikal Malaysia Melaka, Malaysia \\ ${ }^{2,3}$ School of Electrical System Engineering, Universiti Malaysia Perlis, Perlis, Malaysia \\ ${ }^{4}$ School of Computer and .Communication Engineering (SCCE), Universiti Malaysia Perlis, Perlis, Malaysia \\ ${ }^{5}$ Faculty of Electrical Engineering, Universiti Teknikal Malaysia Melaka, Malaysia
}

\begin{tabular}{l}
\hline \hline Article Info \\
\hline Article history: \\
Received Sep 24, 2020 \\
Revised Jan 15, 2021 \\
Accepted Feb 5, 2021 \\
\hline
\end{tabular}

Keywords:

Artificial intelligence

High voltage equipment

Partial discharge

Power cables

Self organizing maps

Statistical features

Transformation

\begin{abstract}
Condition-based monitoring (CBM) has been a vital engineering method to assess high voltage (HV) equipment and power cables conditions or health levels. One of the effective CBM methods is partial discharge (PD) measurement or detection. PD event is the phenomenon that always associated with insulation healthiness. PD has been measured and evaluated in this paper to discriminate PD signals from a good signal. A mixed-signal being fed at an AI technique with statistical modified input data to do fast classification (less than five seconds) with nearly zero error. In this paper, an unsupervised neural network is applied for PD classification. The methods combine the self-organizing maps (SOMs) and feature statistical transformation. By the combination of these methods, the 'range' normalization method produced the best classification outcomes. This development decided that PD information was effectively correlated and grouped by means of MATLAB's SOM Toolbox and transformation device to discriminate the normal signal from the PD signal.
\end{abstract}

This is an open access article under the CC BY-SA license.

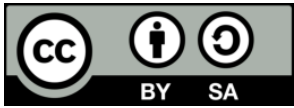

\section{Corresponding Author:}

Zul Hasrizal Bohari

Faculty of Electrical and Electronic Engineering Technology

Universiti Teknikal Malaysia Melaka, Melaka, Malaysia

Email: zulhasrizal@utem.edu.my

\section{INTRODUCTION}

Power utility companies apply a condition based monitoring (CBM) technique for fast failure revelation that are implemented either intrusive or vice versa [1-3]. This CBM procedure is an add-on routine from the regular maintenance that is either preventive corrective or predictive maintenance [4, 5]. The implementation of CBM facilitates engineers with a new descriptive way of understanding the need for different maintenance regimes according to the level of health and ageing of each HV equipment [6-8]. A PD survey is the most accurate way to evaluate on HV apparatus or cable over the live operation [9-13].

PD is a localized current breakdown that is generally found in solid, gaseous, or fluid form interact with void $[1,14,15]$. Where PD has arisen, the transient pulse has been produced for a short time, and this effect is periodic. This difficulty was that, during much of the machinery still being electrified, each on-line system created a sound or noise that eventually compromised the whole collection of PD data and definitely represented a piece of misinformation. The first and the most common technique for classifying PD is presently the assessment of phase resolved PD (PRPD) patterns that widely used by relevant equipment manufacturers $[16,17]$. In the paper, the author proposes a new heuristic method via hybridization of both statistical transformation and artificial neural networks to do clustering between PD and standard data. Self 
organizing maps (SOM) has been widely applied for data mining operations. Basically, SOM operated as a neurological brain towards data clustering or classification task [18-20]. Even though SOM already explored more than couple of decades but the real ability of clustering still reliable and fast comparing to the newly introduced data mining methods available in the research arena. Hence, in the document, an artificial intelligence (AI) namely SOM with pertinent statistical conversion is suggested to hinder this scenario. The system established is seen as a conversion of specialist knowledge to the firmware. Classification results could be considered appropriate but have room for upgraded frameworks.

\section{RESEARCH PROCEDURE}

Few approaches have been used to classify PD activity [21-23]. In this paper, it is focused on using statistical feature transformation and SOM method since these methods have proven excellent performance in other applications such as on load profiling, mechanical integrity detection, and medical assessment.

- Statistical feature transformation

First, the statistical transformation stages are important to have a better interpretation of various linkages among the variables in a issue. By boosting the band of learning variables that portray a given issue, the ability to segregate the data loading also surges.

- Self-organizing map (SOM)

Some studies, such as (Stefanovič \& Kurasova, 2011) and (Vesanto, Himberg, Alhoniemi, \& Parhankangas, 1999), confirmed that now the 'Self-Organization' SOMs are used as a category and foresee the required data that has been organized in low-dimensional neurons [18, 19]. In the meanwhile, 'Maps' means that the feature can be discovered to evaluate the characteristic of input data from the realization mapping. The simple topology used is either rectangular or hexagonal.

\section{METHOD}

Figure 1 shows the research flow for the PD coordination procedure used in this research. In this research, sets of confirmed PD events and typical or normal situations are chosen at first to determine the clustering ability of SOM. The data are mixed up to test the strength of SOM to distinguish without being trained.

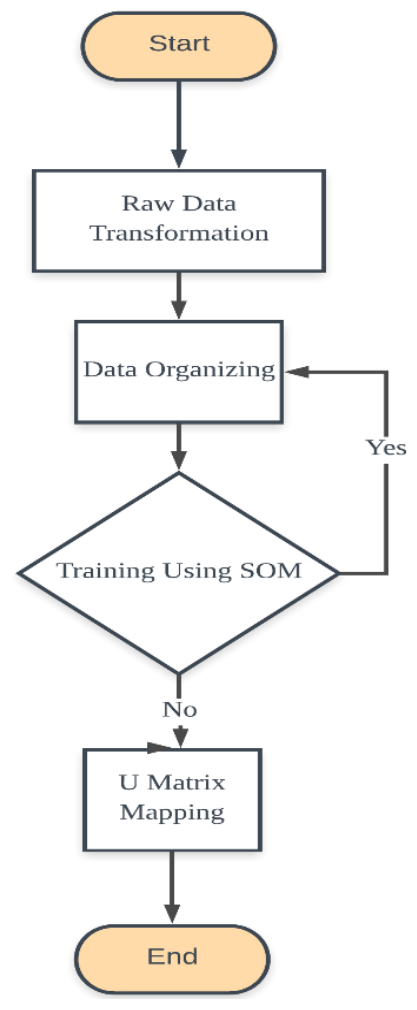

Figure 1. PD classification process 
- References raw feature data transformation

The PD data measured by the equipment, usually only recorded PD data in milliVolt $(\mathrm{mV})$. Therefore, it is not sufficient for MATLAB SOM Toolbox for analyzing and segregation. For SOM classification, at least three types of data needed. Statistical transformation method applied in this step to manipulate the raw data using the charge, $\mathrm{Q}$;

$$
Q=C \times V
$$

where $\mathrm{Q}$ is formed charges, $\mathrm{C}$ refer to the cable capacitance and $\mathrm{V}$ is the magnitude of voltage. Capacitance of cable (XLPE), C is figured thru employing cable capacitance equation for underground;

$$
C=\frac{2 \pi \epsilon_{0} \epsilon_{r}}{\ln \frac{R}{r}}
$$

where $\varepsilon_{0}$ is the permittivity of the agent, $\varepsilon_{r}$ represent free space permittivity, $r$ is the semidiameter of the electrode inside and $\mathrm{R}$ is the sheath radius. Then, the pair of input combined into potential energy, form of $\mathrm{U}$;

$$
U=\frac{Q \times V}{2}
$$

where $\mathrm{Q}$ refers to charge composed, $\mathrm{V}$ is voltage magnitude.

- Input coordination for SOM

SOM used the three sets of primary data from the previous process, which are voltage $(\mathrm{V})$, charges $(\mathrm{Q})$, and potential energy (U) with appropriate data organizing, to be arranged in the MATLAB environment before the classification process even started. The transformed raw data is carefully organized in a suitable mode. The importance of proper data organization will equip SOM with decent data layout to produce a decent classification result. In this study, 33 identified known PD situations and 55 typical scenarios are mixed together in a sample group to validate SOM's ability to self-classify even without being educated as an effective, unsupervised neural network.

- Training using SOM

For this research projects, only selected between 100 and 320 number of neurons are utilized to divide the set of measurement data for preliminary quest. Relevant data that gathered are quantization error, topographic error, training time (sec), and map size. Quantization is by reduction of the output values into manageable sets of value. At the same time, topographic refers to the cluster arrangement of physical mapping ability with appropriate map sizing. All these data will be further evaluated and compared for determining the best result for each normalization method and continue with the U-Matrix mapping investigation.

- U-Matrix mapping

Finally, the U-Matrix mapping study is carried out to check the classifier output graphically. Each map will be detailed analyzed for clustered grouping to conclude on the SOM ability and complete the classification process. Each normalization method typically will have produced a different mapping result that differentiates accordingly.

\section{RESULTS AND DISCUSSION}

After the completion of the data preprocessing, MATLAB SOM will do the final classifying process. The results and inquiry of each classification, especially on the training duration, quantization, and topographic error. The best performance will be mapped to do depth examination on the final classification results. In 4.1, 4.2, 4.3, and 4.4, the analysis and discussion of each classification result are obtainable in the next paragraphs. U-Matrix mapping will only show the dominance PD and standard data label if the input data relatively similar to each other. Each hexagonal will represent more than one data, more data group in each node, hence produce a bigger hexagon.

\section{1. 'Var' normalization}

In Table 1, the best outcomes with 200, 260 and 300 neurons have been seen in the SOM classification. With 200 neurons, one second or more contrast with 2 neurons (260 and 300 neurons) at 4 seconds the quickest time for training is observed. Still, in terms of quantization, 200 neurons produce a relatively low error at 0.001 . Therefore, either 260 or 300 neurons are much more suitable to be selected as 
the better matching neurons for 'var' normalization. After that, all neurons show a fair training period at 4 seconds when examined 300 neurons head by head. But once again, a correct map sizing is required to generate a better classification, and that shows that 300 neurons are the best choice for this 'var' method and that U-Matrix mapping is to be carried out.

Table 1. The collection of 'var' method

\begin{tabular}{ccccc}
\hline \multirow{2}{*}{ No. of neuron } & \multicolumn{4}{c}{ Classification result } \\
\cline { 2 - 5 } & Map size & Quant. error & Topo. error & Train. time (sec) \\
\hline 100 & {$[17,6]$} & 0.021 & 0.068 & 0 \\
120 & {$[17,7]$} & 0.014 & 0.034 & 1 \\
140 & {$[20,7]$} & 0.009 & 0.034 & 1 \\
160 & {$[20,8]$} & 0.003 & 0.011 & 1 \\
180 & {$[23,8]$} & 0.002 & 0.011 & 1 \\
200 & {$[22,9]$} & 0.001 & 0.000 & 1 \\
220 & {$[24,9]$} & 0.001 & 0.011 & 7 \\
240 & {$[27,9]$} & 0.000 & 0.045 & 3 \\
260 & {$[26,10]$} & 0.000 & 0.000 & 4 \\
280 & {$[28,10]$} & 0.000 & 0.080 & 7 \\
$\mathbf{3 0 0}$ & {$[\mathbf{2 7}, \mathbf{1 1}]$} & $\mathbf{0 . 0 0 0}$ & $\mathbf{0 . 0 0 0}$ & $\mathbf{4}$ \\
320 & {$[29,11]$} & 0.000 & 0.000 & 3 \\
\hline
\end{tabular}

In Figure 2(a), the optimum number of neurons (300 neurons) is plotted by U-Matrix mapping. Both PD operations are separated from regular operations in the map. In the diagram, a group of ordinary conditions is divided into three distinct clusters but closed one by the thickness of a black hexagon formed and also the gradient value labeled as N1, N2 and N7. To analyze each component of inputs, the plane representation shown in Figure 2(b) that all three SOM inputs generated a distinct separation (yellow boxes) at the bottom left for all types of data, and this is analogous to the overall mapping result. This mapping result demonstrated that adopting the 'var' method, the classification process is valid with the severe condition lying at the bottom left (red dotted line box) and the good (green dotted line box) at the bottom right.

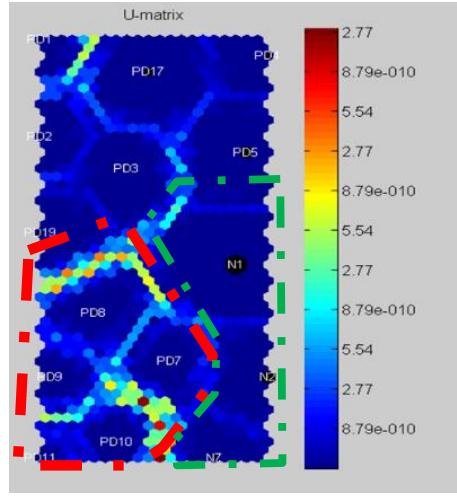

(a)

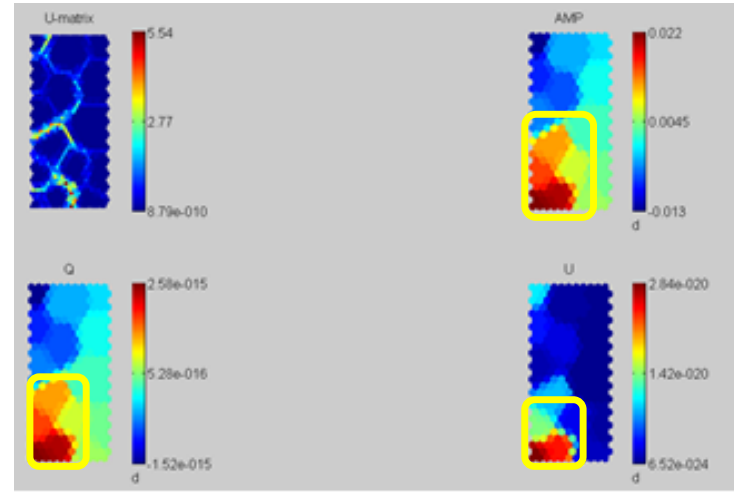

(b)

Figure 2. (a) The mapping outcome for neurons with, (b) Its component mapping

\section{2. 'Range' normalization}

Five neurons display zero for both the quantization error and topographical error in the 'range' standardization approach in Table 2. In order to remove the challenger, the best training time must first be equal to or below 5 seconds, notably for a limited number of data. Consequently neurons of either 300 or 320 were not eligible under these conditions and were left to be tested with only three neuronal values. An advance comparison to judge the map sizing by comparing between these three number of neurons, and based on this judgment, 280 neurons are the most significant map size with $25 \times 11$ volume with 4 seconds training time. That value of the neurons is chosen to be traced via the U-Matrix to validate the outcome of the classification. 
Table 2. The collection of 'range' method

\begin{tabular}{cccccc}
\hline \multirow{2}{*}{ No. of neuron } & \multicolumn{5}{c}{ Classification result } \\
\cline { 2 - 6 } & Map size & Quant. error & Topo. error & Map size & Training time (sec) \\
\hline 100 & {$[17,6]$} & 0.002 & 0.205 & 0.057 & 0 \\
120 & {$[17,7]$} & 0.002 & 0.057 & 0.011 & 1 \\
140 & {$[20,7]$} & 0.002 & 0.057 & 0.159 & 1 \\
160 & {$[20,8]$} & 0.001 & 0.057 & 0.534 & 1 \\
180 & {$[23,8]$} & 0.000 & 0.034 & 0.000 & 2 \\
200 & {$[22,9]$} & 0.000 & 0.000 & 0.000 & 2 \\
220 & {$[24,9]$} & 0.000 & 0.091 & 0.205 & 2 \\
240 & {$[24,10]$} & 0.000 & 0.090 & 0.000 & 3 \\
260 & {$[26,10]$} & 0.000 & 0.148 & 0.534 & 4 \\
$\mathbf{2 8 0}$ & {$[\mathbf{2 5 , 1 1 ]}$} & $\mathbf{0 . 0 0 0}$ & $\mathbf{0 . 0 0 1}$ & $\mathbf{0 . 0 0 0}$ & $\mathbf{4}$ \\
300 & {$[27,11]$} & 0.000 & 0.000 & 0.000 & 5 \\
320 & {$[29,11]$} & 0.000 & 0.466 & 0.000 & 5 \\
\hline
\end{tabular}

The U-Matrix outcome in 'range' scheme of 280 neurons is presented beneath in Figure 3(a). Based on the U-Matrix mapping, the same functional outcome produced similar to the previous method. Both standard data are isolated from PD. However this approach displays that the dividing amid PD and the normal is close and integral with less hexagonal volume, and the separating method is less prevalent compared to ' $v a r$ ' (for N7 and N2). Even at the edge of the standard data, PD7 (orange cycle) is found. When discussing Figure 3(b), all three components show a noteworthy separation of data, especially for current (AMP) and charge (Q) all the standard data move near the bottom right while for energy (U), the standard data grouped in the middle bottom. From the analysis, this might be among the favourable scheme to fix PD classification with a mixed-up mapping figure that might affect the intended classification process. Yet, the separation of severe PD is distinctive and profound in Figure 3(b) (yellow boxes) and maybe the introduction of more features needed to achieve a better classifier result.

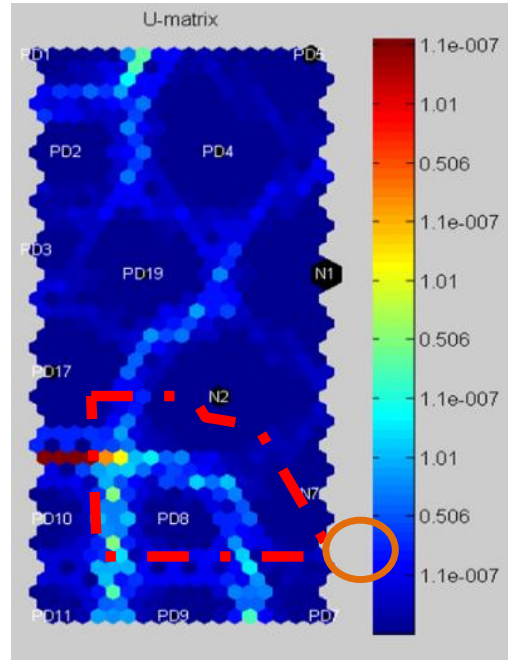

(a)

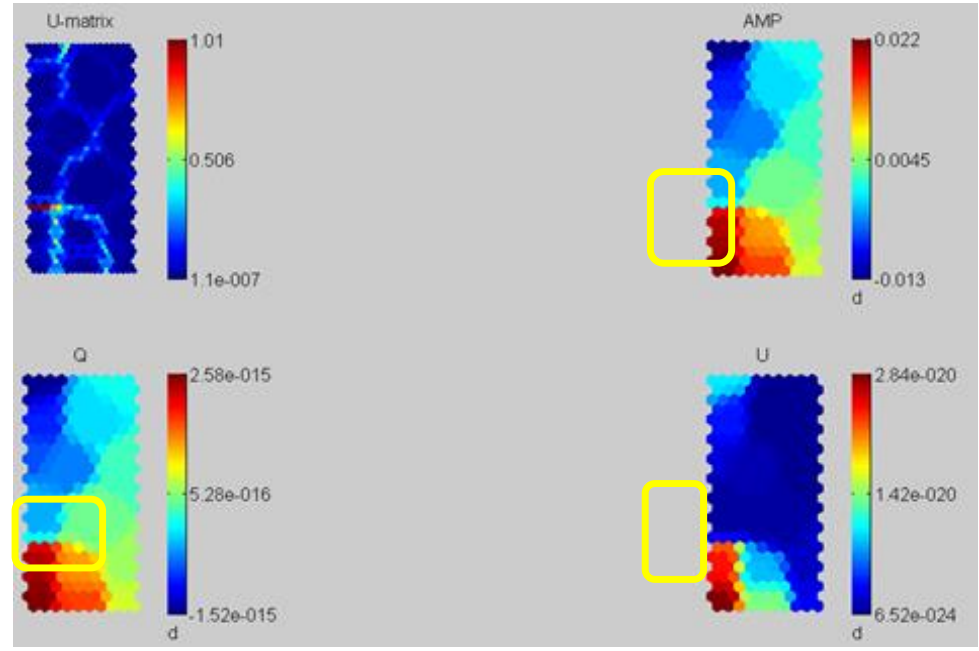

(b)

Figure 3. (a) The mapping outcome for 280 neurons with, (b) Its component mapping

\section{3. “' 'Log' normalization}

The best performance for the 'log' normalization technique achieved by both 200 and 300 neurons, as recorded in Table 3. General, all of the neurons formed zero was recorded aimed at quantization performance. In contrast, for topographic, only two values of neurons achieved zero, while the others the error recorded poor results with more than 0.05 errors. If it occurs, the final pair neurons display a certain rate of training time three seconds) and quantification and topographical error (0.000). First the assessment of the scale of the map will decide the best feasible grouping. The difference is the size of the map seen between two neurons with a stronger map size $(19 x 16)$ of 300 neurons. This importance of the neurons is undertaking an inspection of the U-Matrix for output validation. 
Table 3. The collection of ' $l o g$ ' method

\begin{tabular}{ccccc}
\hline \multirow{2}{*}{ No. of neuron } & \multicolumn{4}{c}{ Classification result } \\
\cline { 2 - 5 } 100 & Map size & Quant. error & Topo. error & Train. time (sec) \\
120 & {$[11,9]$} & 0.000 & 0.205 & 0 \\
140 & {$[12,10]$} & 0.000 & 0.057 & 1 \\
160 & {$[13,11]$} & 0.000 & 0.057 & 2 \\
180 & {$[15,12]$} & 0.000 & 0.057 & 2 \\
200 & {$[15,13]$} & 0.000 & 0.034 & 2 \\
220 & {$[16,14]$} & 0.000 & 0.000 & 3 \\
240 & {$[17,14]$} & 0.000 & 0.091 & 4 \\
260 & {$[17,15]$} & 0.000 & 0.090 & 2 \\
280 & {$[18,16]$} & 0.000 & 0.148 & 3 \\
$\mathbf{3 0 0}$ & {$[\mathbf{1 9}, \mathbf{1 6}]$} & $\mathbf{0 . 0 0 0}$ & 0.386 & 4 \\
320 & {$[19,17]$} & 0.000 & $\mathbf{0 . 0 0 0}$ & $\mathbf{3}$ \\
\end{tabular}

By denoting Figure 4(a), the mapping result showed of assorted mapping. N1 and N2 data grouping (green dotted line box) separated well from the majority PD situation, only N7 (orange circle), where this data alliance is drifted away (orange circle) from the rest. The N7 party is debating the utility of clustering using the ' $\log ^{\prime}$ method. Although, above all, much of the traditional data is also rightly separated into their category of operation. It can be considered almost triumphant because the boundary is precisely in the correct data location. On this map, the red dotted box indicates the most critical PD activity is separated.

In Figure 4(b), a plane representation of the result of the mapping is shown both current (AMP), and charge (Q) successfully divided the severe PD activity data. At the same time, energy (U) data cannot differentiate the normal from the PD data. Therefore, the result for this specific method is unsatisfactory and not fit for PD clustering task. To overcome the setback that happened, the variation of data is needed with additional measurement.

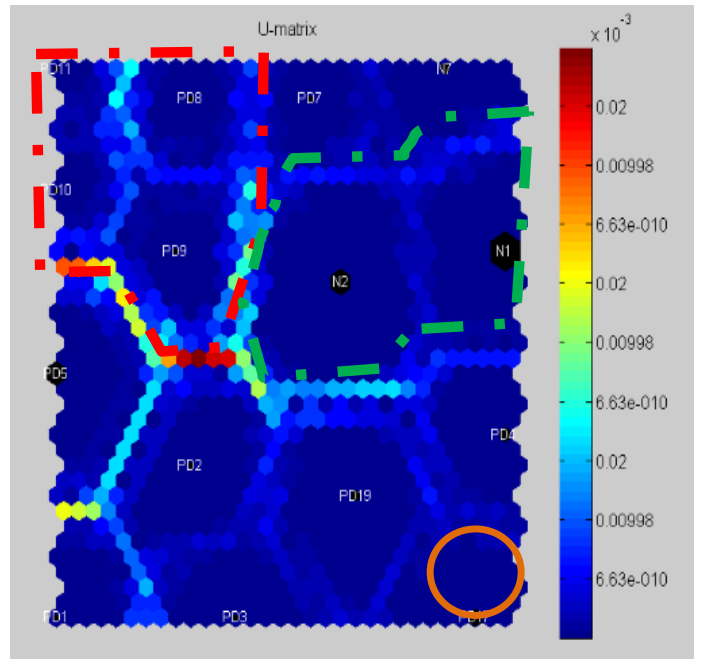

(a)

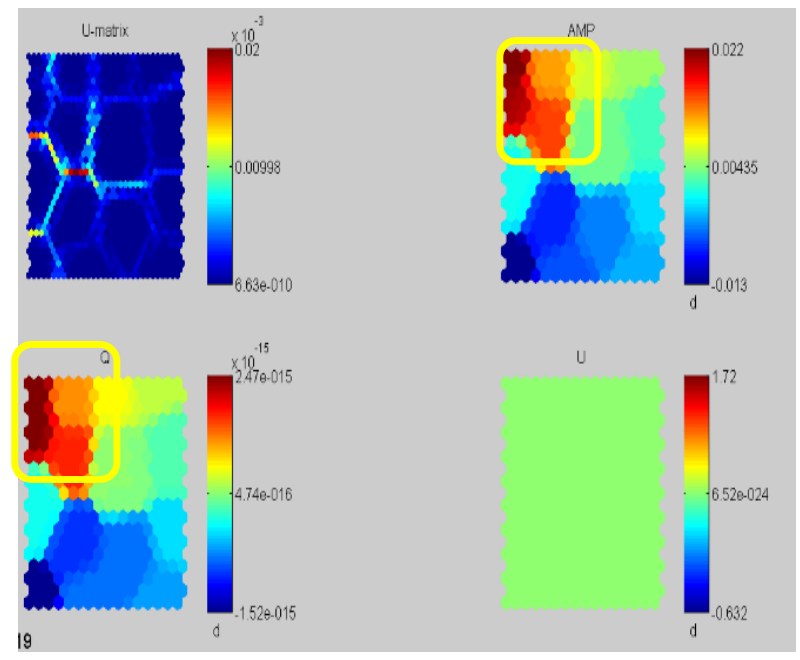

(b)

Figure 4. (a) The mapping outcome for 300 neurons with, (b) Its component mapping

\section{4. ' 'Logistic' Normalization}

Lastly, through the 'logistic' normalization approach as per Table 4, three neurons managed zero error (both quant and topography) at 160, 220, and 280 neurons. Despite the fact, the other neurons value generated rather considerable error exclusively on the topographic portion even with lesser training times. The swift neurons to achieve zero for both errors are at 160 and 220 neurons, but with a slight mapping size. Whereas, for 280 neurons that produced near-zero error, even though the training time is a bit higher, possessed a better mapping size that typically aids for the better clustering map analysis. Later, the rate of 280 neurons is selected for U-Matrix mapping for profundity analysis. 
Table 4. The collection of 'logistic' method

\begin{tabular}{ccccc}
\hline \multirow{2}{*}{ No. of neuron } & \multicolumn{4}{c}{ Classification result } \\
\cline { 2 - 5 } & Map size & Quant. error & Topo. error & Train. time (sec) \\
\hline 100 & {$[17,6]$} & 0.003 & 0.023 & 0 \\
120 & {$[17,7]$} & 0.002 & 0.011 & 1 \\
140 & {$[20,7]$} & 0.001 & 0.034 & 0 \\
160 & {$[20,8]$} & 0.000 & 0.000 & 2 \\
180 & {$[23,8]$} & 0.000 & 0.182 & 6 \\
200 & {$[22,9]$} & 0.000 & 0.386 & 3 \\
220 & {$[24,9]$} & 0.000 & 0.000 & 2 \\
240 & {$[27,9]$} & 0.000 & 0.034 & 4 \\
260 & {$[26,10]$} & 0.000 & 0.568 & 3 \\
$\mathbf{2 8 0}$ & {$[28, \mathbf{1 0}]$} & $\mathbf{0 . 0 0 0}$ & $\mathbf{0 . 0 0 0}$ & $\mathbf{3}$ \\
300 & {$[27,11]$} & 0.000 & 0.386 & 3 \\
320 & {$[29,11]$} & 0.000 & 0.011 & 7 \\
\hline
\end{tabular}

The reference to Figure 5(a), the segregation of normal (green dotted line box) from PD data (red dotted line box) is quite peculiar and indistinguishable. The group N1 is evidence to be reasonably separated from cluster N2, and N7 and the characteristic replicate to the PD events. Even though the output values (training time, quantization, and topographic error) the finest, only through mapping to discriminate the classifier characteristics. In Figure 5(b) a plane representation is shown; the severe PD events are differentiated well for all inputs (yellow boxes). From the graphical mapping result revealing that the 'logistic' way is unsuitable for the PD classification due to the weak grouping of the mapping result. To improve the method clustering performance, it is recommended to add more input data with different feature extraction methods in the future.

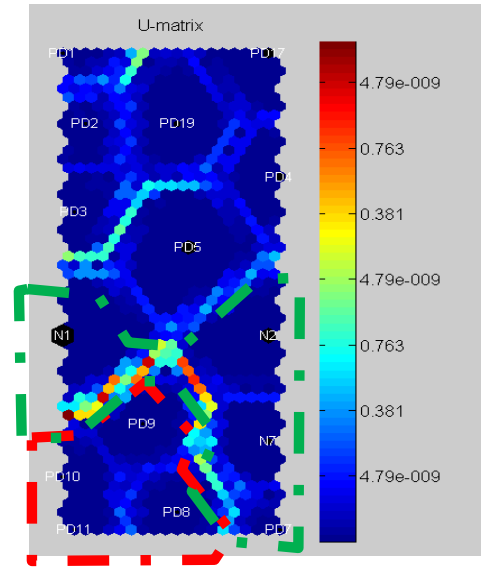

(a)

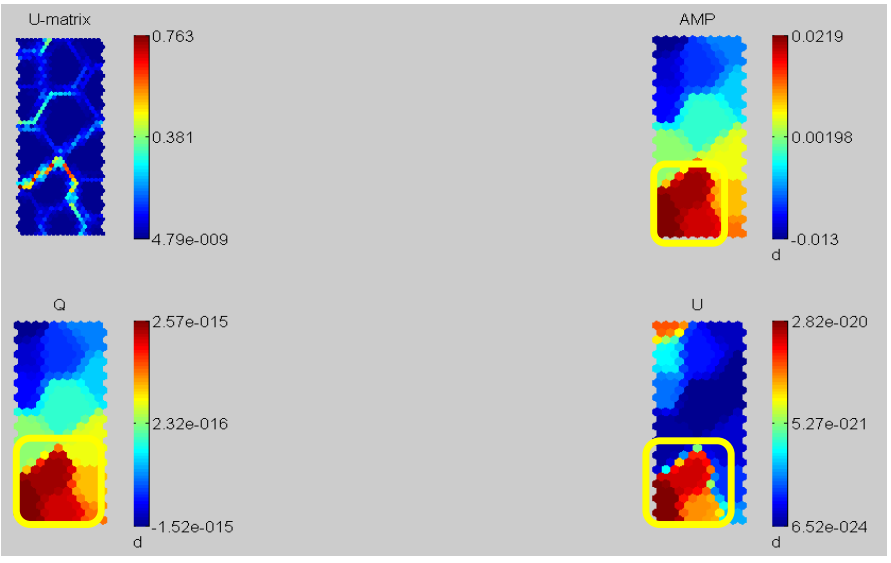

(b)

Figure 5. (a) The mapping outcome for 280 neurons with, (b) Its component mapping

Summarization on the results of the SOM classification as described in Table 5, all the normalization methods achieved the intended objective with a small hiccup. Still, with detailed analysis, at least two ('var,' and 'range' methods) out of four produced are considered near accurate classification and successfully classified and clustered the mixed PD and normal data with an additional excellent severe PD separation. The overall result achieved by this research paper is performing well as near as in the journal [17, 24-26] with different feature transformation method.

Table 5. The summary on SOM classification results

\begin{tabular}{|c|c|c|c|c|c|c|}
\hline $\begin{array}{l}\text { Norm. } \\
\text { method }\end{array}$ & \multicolumn{6}{|c|}{ Classification result } \\
\hline Var & 300 & {$[27,11]$} & $\mathbf{0 . 0 0 0}$ & 0.000 & 4 & Distinct clustering with significant severe PD detected \\
\hline $\log$ & 300 & {$[19,16]$} & 0.000 & 0.000 & 3 & $\begin{array}{c}\text { A normal data mixed up with PD data, unsuitable for } \\
\text { effective PD classification function }\end{array}$ \\
\hline Logistic & 280 & {$[28,10]$} & 0.000 & 0.000 & 3 & $\begin{array}{c}\text { Poor PD and normal data separation, required extended data } \\
\text { features }\end{array}$ \\
\hline
\end{tabular}




\section{CONCLUSION}

Overall, it has been shown that SOM can do smart segregation and classification. The entire normalization methods have victoriously determined the distinctive characteristics of normal and PD cases. Some of the non-specific classification techniques have been some of the aggregate data in the PD group. The prime classification product resolved over ' $v a r$ ' and 'range' normalization with the borderline developed extricates between healthy and PD occurrence. This SOM hybrid of a predictive transformation approach makes it possible for site workers to distinguish smoothly toward PD data without really being exposed to operator error, either due to internally or externally intervention as a PD operation.

\section{ACKNOWLEDGEMENTS}

The authors acknowledge the contribution of Universiti Teknikal Malaysia Melaka, UniMAP, and Ministry of Higher Education Malaysia for the financial support and the resources given through the research grant FRGS/1/2019/TK04UNIMAP/03/5.

\section{REFERENCES}

[1] L. Renforth, A. Burgess, M. Foxall, and T. Raczy, "On-line partial discharge (OLPD) insulation condition monitoring of complete high voltage (HV) networks in the oil \& gas industry," in 2014 IEEE Petroleum and Chemical Industry Conference-Brasil, PCIC Brasil 2014, pp. 14-23, 2014, doi: 10.1109/PCICBRASIL.2014.6968883.

[2] R. Albarracín, J. A. Ardila-Rey, and A. A. Mas'Ud, "On the use of monopole antennas for determining the effect of the enclosure of a power transformer tank in partial discharges electromagnetic propagation," Sensors (Switzerland), vol. 16, no. 2, pp. 1-18, 2016, doi: 10.3390/s16020148.

[3] A. K. S. Jardine, D. Lin, and D. Banjevic, "A review on machinery diagnostics and prognostics implementing condition-based maintenance," Mechanical Systems and Signal Processing, vol. 20, no. 7, pp. 1483-1510, 2006, doi: 10.1016/j.ymssp.2005.09.012.

[4] A. Christina, M. A. Salam, Q. M. Rahman, F. Wen, S. P. Ang, and W. Voon, "Causes of transformer failures and diagnostic methods-A review," Renew. Sustain. Energy Rev., vol. 82, no. July, pp. 1442-1456, 2018, doi: 10.1016/j.rser.2017.05.165.

[5] G. Paoletti and A. Golubev, "Partial discharge theory and applications to electrical systems," IEEE Conf. Rec. Annu. Pulp Pap. Ind. Tech. Conf., pp. 124-138, 1999, doi: 10.1109/papcon.1999.779355.

[6] S. M. Mukhtar, M. Isa, and A. A. Al-Hadi, "Design of UHF antenna sensor for partial discharge detection in high voltage substation," IOP Conf. Ser. Mater. Sci. Eng., vol. 318, no. 1, pp. 1-6, 2018, doi: 10.1088/1757899X/318/1/012052.

[7] M. Arshad and S. M. Islam, "Significance of cellulose power transformer condition assessment," in IEEE Transactions on Dielectrics and Electrical Insulation, vol. 18, no. 5, pp. 15911-1598, 2011, doi: 10.1109/TDEI.2011.6032829.

[8] M. J. Hittel, R. Bingham, and M. K. Sanders, "NFPA 70B recommended practice for electrical equipment maintenance 2002 edition," In 38th IAS Annual Meeting on Conference Record of the Industry Applications Conference, vol. 2, pp. 1280-1284, 2003, doi: 10.1109/ias.2003.1257715.

[9] M. F. Rahman and P. Nirgude, "A new approach to study partial discharge inception due to particles in transformer oil using electric field analysis," 2019 Int. Conf. High Volt. Eng. Technol., ICHVET, pp. 1-6, 2019.

[10] J. Maki, T. Matsuzoe, M. Kozako, M. Hikita, Y. Nakamura, K. Taniguchi, Y. Ikeda, and K. Okamoto "Construction and Verification of Novel Insulation for Next Generation Power Module," 2019 Int. Conf. Electron. Packag., pp. 325-328, 2019.

[11] E. T. Iorkyase, C. Tachtatzis, I. A. Glover, and R. C. Atkinson, "RF-based location of partial discharge sources using received signal features," High Volt., vol. 4, no. 1, pp. 28-32, 2019, doi: 10.1049/hve.2018.5027.

[12] I. A. Soomro and N. Ramdon, "Study on different techniques of partial discharge (PD) detection in power transformers winding: Simulation between paper and EPOXY resin using UHF method," International Journal of Conceptions on Electrical and Electronics Engineering, vol. 2, no. 1, pp. 57-61, 2014.

[13] Y. Luo, Z. Li, and H. Wang, "A review of online partial discharge measurement of large generators," Energies, vol. 10, no. 11. p. 1694, 2017.

[14] Z. Ma, C. Zhou, D. M. Hepburn, and K. Cowan, "Fractal theory based pattern recognition of motor partial discharge," In 2016 Int. Conf. Cond. Monit. Diagnosis, pp. 881-884, 2016, doi: 10.1109/CMD.2016.7757963.

[15] Y. Shibuya, S. Matsumoto, M. Tanaka, H. Muto, and Y. Kaneda, "Electromagnetic waves from partial discharges and their detection using patch antenna," IEEE Trans. Dielectr. Electr. Insul., vol. 17, no. 3, pp. 862-871, 2010, doi: 10.1109/TDEI.2010.5492260.

[16] L. Hao, P. L. Lewin, and S. J. Dodd, "Comparison of support vector machine based partial discharge identification parameters," Conf. Rec. of the 2006 IEEE Int. Symp. Electr. Insul., IEEE, vol. pp. 110-113, 2006, doi: 10.1109/elinsl.2006.1665269.

[17] K. X. Lai, B. T. Phung, and T. R. Blackburn, "Partial discharge analysis using PCA and SOM," In 2007 IEEE Lausanne POWERTECH, Proceedings, pp. 2133-2138, 2007, doi: 10.1109/PCT.2007.4538648.

[18] M. Majumder, "Impact of urbanization on water shortage in face of climatic aberrations: Artificial neural network," 
Spinger, pp. 49-54, 2015.

[19] J. Vesanto and E. Alhoniemi, "Clustering of the self-organizing map," IEEE Transactions on Neural Networks, vol. 11, no. 3, pp. 586-600, May 2000., doi: 10.1109/72.846731.

[20] Z. H. Bohari, M. H. Jali, M. F. Baharom, M. N M.Nasir, H. I. Jaafar, and W. M. Bukhari, "EMG signal statistical features extraction combination performance benchmark using unsupervised neural network for arm rehab device," International Journal of Applied Engineering Research, vol. 9, no. 22, pp. 12393-12402, 2014.

[21] M. Wang, A. J. Vandermaar, and K. D. Srivastava, "Review of condition assessment of power transformers in service," IEEE Electr. Insul. Mag., vol. 18, no. 6, pp. 12-25, 2002, doi: 10.1109/MEI.2002.1161455.

[22] G. Robles, M. Sanchez-Fernandez, R. Albarracin Sanchez, M. V. Rojas-Moreno, E. Rajo-Iglesias, and J. M. Martinez-Tarifa, "Antenna parametrization for the detection of partial discharges," IEEE Trans. Instrum. Meas., vol. 62, no. 5, pp. 932-941, 2012, doi: 10.1109/TIM.2012.2223332.

[23] F. C. Gu, H. C. Chang, F. H. Chen, C. C. Kuo, and C. H. Hsu, "Application of the Hilbert-Huang transform with fractal feature enhancement on partial discharge recognition of power cable joints," IET Sci. Meas. Technol., vol. 6, no. 6, pp. 440-448, 2012, doi: 10.1049/iet-smt.2011.0213.

[24] N. Pattanadech and P. Nimsanong, "Partial discharge classification using principal component analysis combined with self-organizing map," In IEEE Region 10 Annual International Conference, Proceedings/TENCON, pp. 1-5, 2015, doi: 10.1109/TENCON.2014.7022348.

[25] W. J. K. Raymond, H. A. Illias, and A. H. A. Bakar, "Classification of partial discharge measured under different levels of noise contamination," PLoS One, vol. 12, no. 1, 2017, doi: 10.1371/journal.pone.0170111.

[26] W. J. K. Raymond, H. A. Illias, A. H. A. Bakar, and H. Mokhlis, "Partial discharge classifications: Review of recent progress," Measurement: Journal of the International Measurement Confederation. vol. 68, pp. 164-181, 2015, doi: 10.1016/j.measurement.2015.02.032. 